Ce que les émotions font faire aux professionnels du droit:

Jalons pour une approche praxéologique des expressions émotionnelles dans la sphère judiciaire

\title{
Julie Colemans
}

Centre de Recherche et d'Interventions Sociologiques, Université de Liège, Liège, Belgique

\section{Résumé}

L'article propose des pistes de réflexion pour pouvoir saisir la dimension émotionnelle de l'action judiciaire. A partir des acquis de l'ethnométhodologie, il propose d'appréhender les émotions praxéologiquement, c'est-à-dire comme des accomplissements pratiques afin de décrire la manière dont les expressions émotionnelles sont perçues et interprétées publiquement et en situation. Les émotions apparaissent alors comme des appuis pratiques à la coordination et à la compréhension.

\section{Mots-clés}

droit en action, expressions d'émotions, interprétation, perception

\section{Abstract}

This article offers some areas for consideration when seeking to understand the emotional dimensions of legal action. Beginning with the lessons of ethnomethodology, it suggests grasping emotions praxeologically, that is, as a practical accomplishment, in order to describe how emotional expressions are perceived and interpreted publicly and in context. Emotions thus appear as practical supports for coordination and understanding in interactional sequences.

\section{Keywords}

emotions, interpretation, law, perception, sensitive action

\footnotetext{
Corresponding author:

Julie Colemans, Centre de Recherche et d'Interventions Sociologiques, Université de Liège, 3 Place des Orateurs, B 4000 Liège, Belgique.

Email: julie.colemans@ulg.ac.be
} 
'Iram non novit jus' ${ }^{1}$ est le titre d'un des rares articles francophones qui se penche sur le statut des émotions dans le raisonnement juridique. En associant droit et émotions, Alain Papaux (2009) interroge le modèle juspositiviste et sa formalisation du raisonnement juridique (Kelsen, 1999). La doctrine juridique présente classiquement le droit à travers le prisme de la rationalité, rejetant par là-même les émotions en dehors de la sphère juridique. La justice doit agir les yeux bandés. Le juge doit accomplir sa mission dans une tempérance qu'aucune circonstance ne peut rompre. L'office du juge est dès lors guidé par un impératif d'impartialité et d'objectivité, ce qui est censé tenir les émotions loin du jugement juridique.

Malgré la rigueur des principes rappelés par la doctrine, le droit tel qu'il se pratique ne peut se satisfaire des catégories étanches qui l'enferment dans des espaces régis par la raison, laissant par conséquent les émotions en dehors du phénomène juridique. Il apparaît pourtant que les émotions sont de plus en plus prégnantes dans les textes législatifs ${ }^{2}$ et deviennent incontournables dans les activités judiciaires. Des travaux ont d'ailleurs étudié la rhétorique émotionnelle dans les prétoires (Barbot \& Dodier, 2014; Lynch \& Haney, 2014). Ces approches mettent en avant la manière dont sont instrumentalisées les émotions plaidées afin d'influencer l'issue du procès.

Cette contribution interroge la place des expressions émotionnelles dans l'activité judiciaire. On discutera la possibilité d'affiner notre compréhension du droit, et particulièrement du droit en action, grâce à la prise en compte de sa dimension émotionnelle. Il y a un intérêt sociologique à ne pas évacuer d'emblée la question des émotions au profit d'une vision rationaliste de l'action. Pour ce faire, on propose d'étudier les émotions et le droit dans une perspective praxéologique. ${ }^{3}$

Saisir praxéologiquement les émotions, c'est-à-dire les appréhender comme des accomplissements pratiques, permet d'en préserver l'épaisseur phénoménologique. On ne propose pas une théorie, des concepts et des catégories sur les émotions, mais une manière non-mentaliste d'étudier les émotions en contexte. Très concrètement, l'enjeu est d'offrir un cadre analytique permettant de décrire les manières dont se manifestent les émotions dans l'interaction, la façon dont elles sont perçues et interprétées publiquement et en situation, afin de montrer comment elles participent à l'action judiciaire en train de se faire et comment elles sont des appuis pratiques à la compréhension et à la coordination. Il s'agit donc de comprendre ce que les émotions font faire aux professionnels du droit dans des environnements concrets participant à la définition de l'action.

Pour ce faire, nous préciserons dans un premier temps la posture de recherche proposée. Plutôt que de s'intéresser aux émotions éprouvées par les acteurs, nous nous focaliserons sur la manière dont les expressions émotionnelles sont perçues et interprétées, proposant de saisir le phénomène émotionnel 'à la deuxième personne'. Ensuite, nous verrons comment les expressions émotionnelles sont perçues et orientent les cours d'action. Enfin, nous discuterons des pistes méthodologiques pour mettre en œuvre ce programme de recherche.

Les pistes et réflexions proposées dans cet article s'appuient sur 15 observations d'audiences au tribunal de la famille et au tribunal du travail en région liégeoise (Belgique) ainsi que sur 10 entretiens avec des magistrats ( 3 auditeurs, 4 juges du tribunal du travail et 3 juges du tribunal de la famille) et 5 entretiens exploratoires avec des avocats. 


\section{Saisir les émotions 'à la deuxième personne'}

\section{L'approche praxéologique des émotions}

Les directions qu'a prises la littérature sociologique à propos des émotions montrent à quel point le phénomène émotionnel est complexe. Les conceptualisations qui en sont proposées peinent à rendre compte de cette complexité, son appréhension étant tributaire des aspects sélectionnés par le chercheur qui les juge pertinents (Turner, 2009). La démarche qui consiste à vouloir définir et objectiver les émotions à partir des catégories scientifiques surplombantes semble perdre ce qui fait la spécificité du phénomène émotionnel (Game, 1997).

Afin d'éviter l'écueil des approches scientifiques qui visent à objectiver le phénomène émotionnel, nous partirons des acquis de l'ethnométhodologie qui parait être la plus à apte à rendre compte des manières dont les expressions émotionnelles interviennent dans les activités des professionnels du droit. La compréhension du phénomène émotionnel passe alors moins par la définition de son objet que par la description du rôle que jouent les émotions dans l'interaction et dans le raisonnement juridique pratique. Cela implique d'adopter le point de vue des juges et avocats, non de l'observateur extérieur. Le sociologue utilise alors les catégories pratiques des professionnels du droit afin d'exposer les manières dont les émotions interviennent dans l'activité judiciaire. Cette manière d'appréhender praxéologiquement les émotions de manière contextualisée et non mentaliste permet d'en conserver sa dimension expérientielle.

Le fondateur de ce courant de recherche a d'ailleurs évoqué la question des émotions dans ses recherches en ethnométhodologie. Garfinkel (2007: 115) y soulignait le rôle que joue la compréhension d'arrière-plan dans la production, le contrôle et la reconnaissance des 'affects sociaux' (Garfinkel, 2007: 115). Partant de ses enseignements sur le socle routinier des activités ordinaires, nous faisons l'hypothèse que l'expression des émotions appartient à ce savoir tacite partagé sur les propriétés d'arrière-plan des scènes quotidiennes et participe à l'intercompréhension. Malgré et en surtout en raison de leur naturalité, les expressions émotionnelles sont peu présentes dans les comptes rendus sociologiques car elles sont vues sans qu'on y prête attention ('seen but unnoticed').

Le rôle des expressions émotionnelles dans la structuration des activités a été mis en exergue grâce à plusieurs expériences menées par Garfinkel. Il a d'une part montré à travers les 'behaviorized descriptions' à quel point les connaissances de sens commun des structures sociales sont nécessaires pour la compréhension des activités. Ces descriptions devaient être totalement objectives et ne pas mentionner les émotions, les événements biographiques, les connaissances du quotidien, les motivations, les éléments personnels. Cette amnésie en ce qui concerne le sens commun des structures sociales empêche de rendre compte de la manière dont se déroulent réellement les activités. Il a d'autre part mis en place des 'breaching experiments', qui créaient des situations d'action dans lesquelles un des partenaires était totalement désengagé: les étudiants expérimentateurs devaient interagir avec les membres de leur famille comme s'ils étaient des étrangers. Cette prise de distance a provoqué de vives réactions émotives de la part de leur entourage.

Ces expériences montrent à quel point l'engagement dans l'interaction qui se manifeste à travers l'expression émotionnelle est lié à des attentes d'arrière-plan (Garfinkel, 2007: 115-119) qui permettent et mettent en œuvre la compréhension de l'action. L'implication 
émotionnelle dans certains types d'interaction fait partie des attentes d'arrière-plan, ces attentes étant ce qui confère aux scènes de la vie quotidienne leur caractère banal et familier (Garfinkel, 2007: 100).

Voyons à présent comment prolonger ces enseignements pour saisir les émotions dans la sphère judiciaire.

\section{Les émotions à la deuxième personne}

Patricia Paperman est l'une des pionnières dans le champ de la sociologie francophone des émotions. Dans une perspective phénoménologique et interactionniste, elle propose de s'intéresser aux façons dont les 'émotions sont reconnues, étiquetées, évaluées, gérées, exprimées’ (Paperman, 1995: 11), ce qui contribue à façonner le phénomène luimême. Il s'agit non pas d'étudier les émotions en tant qu'états internes, mais de replacer ces expressions émotionnelles dans le contexte de leur énonciation afin de mettre en exergue leur rôle dans notre compréhension de la vie quotidienne. 'L'analyse des émotions s'efforce dans ce sens de montrer que l'identification des émotions est toujours produite dans le cadre d'une compréhension située' (Paperman, 1995: 17). Elle propose d'appréhender les émotions 'à la troisième personne', c'est-à-dire comme des phénomènes socialement construits. Les émotions ne sont pas attachées à la personne qui les ressent, mais à l'action en train de se faire.

L'analyse en troisième personne aborde la question de l'identification des émotions d'un point de vue que l'on pourrait dire 'externe': elle peut se focaliser par exemple sur un ensemble ou une configuration plus large comprenant les circonstances, les procédures de l'identification, les éléments significatifs de la scène d'action ou d'interaction en cours, ensemble dont l'agencement permet de 'fixer' l'émotion sur l'une des parties de la scène (moment, personne, événement cristallisant l'émotion) (Paperman, 1995: 12).

Patricia Paperman a élaboré une démarche de recherche innovante en externalisant un phénomène réputé intime et subjectif, permettant par là-même à la sociologie de se saisir d'un phénomène laissé traditionnellement aux sciences psychologiques. Nous nous inscrivons dans cette manière d'appréhender le phénomène sensible de manière non-mentaliste en y apportant cependant une nuance épistémologique. La posture adoptée par Paperman apparaît relativement positiviste, plaçant le sociologue en observateur extérieur.

Pour comprendre l'action judiciaire en train de se faire, nous préférons adopter la posture proposée par Ogien - dont il revendique la filiation à l'égard de ce qu'il appelle l'interactionnisme réaliste de Goffman et à l'ethnométhodologie (Ogien, 2007: 104; 2009: 506 ) - qu'il appelle la 'sociologie à la seconde personne' (Ogien, 2009). Le 'tu impersonnel' incarne 'l'alteractant', c'est-à-dire non pas le 'je' qui agit en tant qu'acteur rationnel ou narrateur à la première personne, mais le partenaire dans l'interaction.

Lorsque les émotions sont étudiées du point de vue de celui qui les ressent - les émotions 'à la première personne' - cela mène souvent le chercheur à déterminer dans quelle mesure l'émotion génère l'action (Bandes, 1999; Scherer, 2011). Les émotions ressenties par les acteurs interviennent alors comme moteur de l'action ${ }^{5}$ en ce qu'elles opèrent à un niveau cognitif ou délibératif. Une émotion est ressentie, qu'elle ait une fonction informative ou morale et évaluative (Ben-Ze'ev, 1997; Karstedt, 2002; Paperman, 2000; Pizzaro, 2000), et aide l'acteur à prendre une décision. Ainsi, la honte (Karstedt, 2002), 
l'empathie (Bandes, 1996), la colère, la peur (Cislaru, 2008) ou le dégoût ont été étudiés dans les processus décisionnels ou dans le rapport des individus aux normes juridiques (Flückiger, 2009). La nature cognitive et évaluative des émotions a d'ailleurs été largement reconnue (Damasio, 1995; Nussbaum, 1995; Scherer, 2011) dans le chef de l'acteur qui l'éprouve, dans un schéma causal où la cognition entraîne l'action. Cela a conduit certains chercheurs à se pencher sur la manière dont elles peuvent être régulées (Gross, 2013) et à mettre en avant les règles de sentiments qui sous-tendent l'adéquation des sentiments ressentis face à la situation vécue (Hochschild, 2003).

Dans le champ du droit, cela revient à se demander d'une part comment les émotions influencent le jugement - un juge ressent de la tristesse, de l'indignation ou de la colère, et il se montre par conséquent plus sévère ou, à l'inverse, il éprouve de l'empathie et se montre plus clément - et d'autre part comment le juge parvient à réguler ses émotions.

En renversant la perspective et en passant des émotions ressenties, 'à la première personne', aux émotions perçues, 'à la deuxième personne', le statut des émotions change. En sortant du domaine de l'intime, les émotions exprimées acquièrent un caractère public (Watson, 1995). Elles sont moins attachées à une personne qu'à une situation (Ogien \& Paperman, 1995). On soutient que les expressions émotionnelles perçues dans l'interaction constituent une composante de l'action en train de se faire en ce qu'elles orientent les pratiques de l'interactant qui les observe.

Lors des audiences, les professionnels du droit repèrent et se servent des expressions émotionnelles des participants à l'interaction pour orienter leurs propres pratiques et s'ajuster en situation. Se trouvent au cœur du phénomène observé les émotions exprimées dans l'interaction en tant qu'elles sont perçues et interprétées par les avocats et les juges lors des audiences. Arrêtons-nous à présent sur les activités perceptives et interprétatives afin de voir comment elles participent à l'action judiciaire en train de se faire.

\section{La perception des expressions émotionnelles}

Vouloir saisir les perceptions ${ }^{6}$ oblige le chercheur à se pencher sur la place du corps dans l'action et dans les comptes rendus sociologiques. 'La plupart des théories de l'action ne font aucune place au corps. La sociologie présuppose d'ordinaire l'existence du corps comme siège factuel de l'agir, mais par une sorte de pruderie théorique ne s'y intéresse pas davantage' (Joas, 2001: 34). Comme le fait remarquer Joas, le corps, dans les théories classiques de l'action, ${ }^{7}$ joue uniquement un rôle d'instrument au service de la pure intentionnalité. ${ }^{8}$ On fait l'hypothèse que la compréhension de l'action gagne à ne pas être réduite à une interprétation intellectuelle mais qu'elle passe également par une prise en compte du matériau accessible aux sens (Quéré, 1999: 301). Nous proposons dès lors, à la suite de Dupret et Ferrié (2006), de saisir le corps dans le contexte de ses performances pratiques à travers les activités perceptives, d'une part, et à travers les expressions corporelles qui sont perçues, d'autre part.

\section{Les expressions émotionnelles}

Perceptibles dans l'interaction, les expressions émotionnelles s'incarnent à travers les mouvements du corps, qu'il s'agisse de mimiques faciales, de postures, de déplacements. 
Ces expressions corporelles sont saisies dans l'interaction tout comme le sont le volume de la voix, son débit, les intonations ou encore le jeu du regard (Le Breton, 2004: 46). Lors des audiences, langage discursif et expressions émotionnelles contribuent à la compréhension de l'action en train de se faire en s'éclairant mutuellement (Garfinkel, 2007). Ils informent le discours et le nuancent (Ogien \& Paperman, 1995).

Le regard apparaît comme un guide lors de l'interaction; il peut se montrer sévère, fuyant, courroucé, bienveillant, dubitatif ou moqueur. Un visage grave, des yeux attendris, une voix qui se fait forte, claquante ou au contraire douce ou mal assurée, une posture qui trahit le malaise ou l'assurance, ces expressions incarnées sont directement perçues. Ces signes corporels colorent le discours, offrant de la sorte des clés d'interprétation contextuelles aux personnes qui les perçoivent.

'Poursuivez, Maître.' Cette phrase maintes fois prononcée par le juge lors des audiences peut avoir plusieurs sens. Elle peut être comprise comme une invitation à poursuivre et à développer l'idée exposée - 'Poursuivez, Maître' - ou comme une exhortation à en finir avec l'argument pour passer à un autre point de la plaidoirie - 'Poursuivez, Maître!' Pour l'interpréter, l'avocat utilise les indices non-verbaux qui entourent son énonciation telle que l'intonation, les expressions du visage ou encore la gestuelle.

Un sourcil qui se lève peut marquer le scepticisme ou l'incrédulité de l'interlocuteur. Un même mouvement, un même geste revêt une signification différente selon le contexte d'action. Un sourire peut être perçu comme narquois, sarcastique ou empathique selon le contenu de ce qui se dit et selon sa place dans la séquence d'interaction. Un hochement de tête peut être interprété de différentes manières: soit comme un signe d'approbation, soit comme un signe d'attention, soit encore comme un encouragement à poursuivre (Le Breton, 2004: 62). Cela signifie que tout comme le discours ne trouve de sens en dehors de son contexte d'énonciation, le langage non-verbal a également des propriétés indexicales. ${ }^{9}$ Le corollaire méthodologique d'un tel positionnement implique une approche écologique des émotions. Celles-ci ne peuvent, dans cette perspective, être analysées qu'en situation. Le contexte de leur émergence permet en effet de les comprendre, de leur donner un sens.

\section{Percevoir les expressions d'émotion}

Les activités perceptives impliquent les sens et renvoient aux activités de voir, de regarder, de remarquer, de fixer, de lire, d'être attentif ou 'd'écouter d'une oreille'. Parvenir à rendre compte de ces activités sensibles permet de comprendre comment les expressions émotionnelles sont visibles, pertinentes, sélectionnées et rendue significatives ${ }^{10}$ par les professionnels du droit.

Ainsi, lors des audiences, les juges remarquent ${ }^{11}$ (Coulter \& Parsons, 2002: 213) certaines expressions émotionnelles qui deviennent signifiantes dans le contexte particulier de l'affaire à trancher. Si l'action de 'remarquer' fait appel à la vue, comme l'action de 'voir' ou de 'regarder', son utilisation n'est pas anodine. Ce terme met l'accent sur un événement accessoire, inattendu ou en tous cas, non planifié. Pendant les audiences, alors que le magistrat 'regarde' les parties - son attention est donc dirigée intentionnellement vers les parties - il 'remarque' le mutisme du justiciable, sa colère sourde, son appréhension ... tous termes qui permettent de catégoriser les personnes de manière sensible 
et incarnée. Il ne s'agit pas de catégories légales qui permettraient de qualifier les faits mais de catégories qui renvoient au savoir de sens commun sur les manières de se tenir, de s'exprimer, de rire ou de pleurer ....

Lors d'une audience qui concernait le licenciement d'un travailleur protégé, le travailleur se tenait prostré sur son banc. Il n'a à aucun moment regardé le tribunal mais fixait les pieds du greffier, le regard perdu dans le vide. Après les plaidoiries des avocats, le président lui donna la parole, lui demandant s'il souhaite ajouter quelque chose. Celui-ci se lève et explique que l'entreprise ne l'a jamais apprécié et qu'il était le premier à être mis en chômage technique. Il dit que c'est sans doute à cause de son statut de travailleur syndiqué. L'homme âgé d'une grosse cinquantaine d'année tremble légèrement. Par la suite, le débriefing avec le magistrat nous apprendra que son avocat le décrivait dans ses conclusions comme une personne impulsive et violente ayant agi sous le coup de la colère et ce, afin de justifier son attitude à l'égard de son employeur. Le fait que le travailleur soit resté calme et impassible durant l'audience a amené le magistrat à mettre en doute la thèse soutenue par son avocat. L'employeur a d'ailleurs reçu l'aval du tribunal pour le licencier.

Le caractère remarquable de certains détails peut d'ailleurs faire écho à des impressions et interrogations qui ont éveillé la curiosité du magistrat durant la préparation du dossier. Celui-ci se rend alors plus attentif à certains éléments qui pourraient valider ou invalider une thèse ou authentifier ou décrédibiliser une pièce du dossier. Les indices corporels et les émotions exprimées durant l'audience fournissent des informations supplémentaires au juge, lui permettant de donner de la matière à des documents désincarnés.

\section{Les expressions émotionnelles comme appuis pratiques}

Nous venons de voir comment les expressions émotionnelles s'ancrent dans les mouvements du corps et font l'objet des activités perceptives des magistrats. Nous envisagerons à présent comment elles constituent des appuis pratiques pour les professionnels du droit. Les expressions émotionnelles offrent à la fois des prises (Bessy \& Château-Reynaud, 1995) pour la coordination lors des audiences ainsi que des appuis pour l'interprétation du litige dans le processus décisionnel.

\section{Les expressions corporelles comme appui à la coordination}

Les expressions émotionnelles repérées chez les magistrats, les avocats et les parties présentes à l'audience permettent aux professionnels du droit de s'ajuster lors des audiences.

En étudiant les manières dont les avocats perçoivent les émotions du juge - et de leurs confrères - et s'y ajustent en situation, on peut mieux comprendre la dynamique des plaidoiries. Avant l'audience, l'avocat 'prépare son dossier': il relit ses conclusions et celles de son adversaire, prépare ses pièces et sélectionne les éléments qu'il souhaite mettre en avant oralement. Toutefois, l'activité de plaider est une activité située qui se construit dans l'interaction avec les différents acteurs présents. Ce que l'avocat avait prévu de dire peut être complètement adapté à ce que son adversaire vient de plaider ou suite aux réactions du juge. En outre, pendant qu'il expose ses arguments, l'avocat est 
attentif aux expressions émotionnelles du juge. Ainsi, il convient de saisir comment les avocats repèrent et interprètent les réactions des juges, leurs mimiques, leurs questions, leur posture, et comment ils s'ajustent à ces manifestations émotionnelles. On pense au cas rapporté par un avocat qui, en plaidant, s'aperçoit que le juge regarde par la fenêtre. Il analyse le regard perdu du juge comme le signe que ce dernier 'a décroché de la plaidoirie'. L'avocat décide alors de passer à autre argument de droit qu'il espère plus percutant. A contrario, l'avocat qui sent l'attention accrue du juge insistera sur l'élément qui fait mouche. Un acquiescement, un signe d'énervement ou d'impatience, un regard soutenu, nourrissent les échanges et colorent le discours.

Lorsque le client accompagne son avocat lors de l'audience, ce dernier doit pouvoir le 'maîtriser'. Le juge s'attend à ce que l'avocat gère son client pour éviter tout débordement. Ainsi, lors d'une audience au tribunal de la famille, un avocat d'une quarantaine d'années plaide le dossier de son client, un homme jeune et très nerveux. Lorsque la juge lui pose une question relative à ses revenus, le jeune homme parle fort, explique qu'il est ouvrier en bâtiments et qu'il a peu de revenus. Il évoque notamment les cas de chômage dû aux intempéries. L'avocate de la partie adverse coupe la parole du jeune homme pour attirer l'attention du juge sur le fait qu'il a également des revenus locatifs puisqu'il met en location une maison et un garage. A ce moment, les choses dégénèrent et le jeune homme explique qu'il a des problèmes d'arriéré de loyers et ajoute en se tournant vers son ex-femme que c'est de sa faute puisque la locataire est une de ses amies. Le juge se met alors à fixer intensément l'avocat du jeune homme, qui comprend alors qu'il doit le faire taire. L'avocat pose alors sa main sur l'épaule droite de son client qui se rassied sur le banc et se tait.

Les magistrats et les avocats perçoivent directement la signification des expressions émotionnelles sans devoir procéder à des interprétations. Ils voient la tristesse d'une partie, le malaise des avocats ou l'impatience d'un magistrat. Ils utilisent à la fois le contexte et leur expérience professionnelle pour déchiffrer ce que l'autre vit, ressent, éprouve, 'veut dire', à la seule vue de tels indices corporels même infinitésimaux. Ces expressions émotionnelles huilent les rouages de l'interaction.

\section{Les indices corporels comme appui au raisonnement juridique pratique}

En plus d'être des appuis à la coordination lors des audiences, la perception des expressions émotionnelles et des indices corporels permet également aux professionnels du droit, en particulier aux magistrats, de construire leur décision.

La procédure civile est essentiellement écrite. Les arguments juridiques soutenus par les parties doivent être rédigés dans des conclusions et s'appuyer sur des dispositions législatives. Les faits qui sous-tendent le raisonnement juridique doivent reposer sur des preuves écrites consignées dans un dossier de pièces. Si les magistrats se forgent une idée à la lecture du dossier, les audiences leur permettent de confirmer cette impression, de poser des questions et éventuellement d'infléchir leur jugement. La présence des parties est particulièrement appréciée lorsque les magistrats hésitent, les faits n'étant pas clairement établis. Les magistrats confrontent alors les imputations avancées par les avocats dans les conclusions écrites aux signes qui ressortent de la posture et des discours des parties. 
Un cas de droit social nous a été rapporté par un auditeur. Il permet de comprendre comment la perception du justiciable peut guider l'interprétation du juge. Il s'agissait d'une femme qui était en litige avec le $\mathrm{CPAS}^{12}$ de sa commune. La femme intente une procédure contre le CPAS, qui lui refuse un revenu d'intégration auquel elle prétend avoir droit.

C'était une dame elle avait un problème de revenu. Le CPAS considérait qu'elle n'était pas très claire d'une part en ce qui concerne ses rentrées financières parce qu'elle avait plusieurs comptes, etc., et d'autre part en ce qui concerne une éventuelle cohabitation avec quelqu'un. Dans le dossier, on voyait bien que l'assistante sociale - parce que malgré tout ce sont des personnes aussi qui rédigent les rapports - et donc l'assistante sociale avait des difficultés avec la dame. Les assistants sociaux, ce qu'ils font c'est qu'ils notent aussi ce que les gens disent même s'ils disent des choses méchantes sur eux. Donc par exemple, la personne se montre agressive, elle considère que je ne peux pas rentrer dans son intimité en examinant ses extraits de compte. Et donc il y avait un très gros conflit entre l'assistante sociale et la personne. Et à l'audience, c'est vrai que j'avais lu qu'elle avait un problème de santé, mais, pff, je ne savais même plus ce que c'était et à l'audience, on a vu une dame très très diminuée physiquement, très maigre, en pleurs et c'est vrai que ça apportait quand même du crédit à sa situation qui était de dire qu'elle ne voulait pas frauder mais qu'elle avait une personne qui l'aidait parce que sa maladie faisait qu'elle avait de telles douleurs qu'elle ne savait pas se déplacer toute seule, qu'elle ne savait pas s'assumer toute seule, mais elle ne cohabitait pas au sens d'une cohabitation économique. Donc c'est vrai que ça avait eu un impact là-dessus. A la fin de l'audience, on s'est dit: 'ben cette petite madame, ça colore de manière tout à fait différente ce qu'on a pu lire dans le dossier'. Ici je me suis quand même dit parce que l'assistante sociale notait ce que madame n'aimait pas qu'on lui dise comme remarque. Et l'assistante sociale lui fait une remarque sur la cohabitation et la dame lui répond mais non, je ne cohabite pas et puis vous vous comportez comme si ma couleur de peau avait un impact. Après coup on se dit qu'il y a un conflit entre l'assistante sociale et la dame et que l'assistante sociale n'est plus du tout objective dans ce qu'elle écrit et ce qu'elle fait. (Auditeur tribunal du travail de Liège)

Selon les termes de l'auditeur, il 'voit' une 'dame', 'malade, diminuée physiquement, en pleurs'. La perception de ces signes sensibles lors de l'audience 'colore' le dossier, apportant du crédit aux allégations de la dame. Suite à l'audience, la thèse selon laquelle la dame était une fraudeuse n'est pas retenue. Cet extrait illustre la prégnance des indices sensibles et incarnés dans le processus décisionnel.

On peut appréhender le processus décisionnel comme un long processus interprétatif $^{14}$ qui dépasse le cadre de l'audience. A la lecture des pièces du dossier et des conclusions, le magistrat formule des conjectures et des hypothèses sur le cas à trancher (Quéré, 1999). Ce schéma d'interprétation servira par la suite à interpréter les nouvelles informations tout comme les signes corporels et émotionnels perçus lors des plaidoiries. Certains détails seront alors jugés juridiquement pertinents à l'aune de ce schéma d'interprétation toujours révisable.

Le cas exposé ci-dessus n'avait pas débouché en première lecture sur un schéma interprétatif clair pour le magistrat. Par contre, l'audience fournit des indices qui documentent la thèse de la maladie. Catégorisée comme une personne malade ayant besoin d'aide, la femme ne sera de facto pas qualifiée juridiquement comme une fraudeuse. 
Ainsi pour qualifier un fait, les magistrats peuvent s'appuyer sur des documents du dossier, les conclusions mais aussi sur des signes sensibles perçus lors des audiences qui renvoient à des typifications et à un savoir de sens commun. Qualification juridique et catégorisation ordinaire s'interpénètrent dans le déroulement même des activités judiciaires (Sudnow, 1965).

Les expressions émotionnelles et les indices corporels que le juge perçoit chez les participants au procès permettent d'interpréter certaines pièces du dossier, de renforcer une impression sur l'issue du litige ou d'infléchir une qualification juridique. Elles apparaissent comme des indices qui documentent une thèse et nourrissent leur raisonnement pratique. Selon que le magistrat prépare un projet de jugement ou non, sa perception des plaidoiries variera. Par ailleurs, à la lecture du dossier, certaines pièces ou certains faits demandent un supplément d'explication, qui réside la plupart du temps dans la singularisation du cas. L'audience et son lot d'émotions et de matériau sensible permettent d'humaniser le conflit en singularisant les faits et en les ancrant dans une réalité plus tangible, rendant le cas plus décidable.

\section{Une posture non-ironique ${ }^{15}$}

Une approche praxéologique des émotions implique un positionnement épistémologique non-ironique ${ }^{16}$ (Dupret, 2006; Travers, 2001) hérité de l'ethnométhodologie. Il s'agit de décrire 'comment les membres ordinaires de la société comprennent leurs propres actions, plutôt qu'à ironiser à ce sujet' (Travers, 2001: 352). En refusant d'adopter un point de vue objectivant sur les phénomènes étudiés, cette approche utilise les catégories pratiques des acteurs afin de rendre compte de leur point de vue ordinaire, en mettant en avant leurs procédures d'interprétation et leurs méthodes de travail situées. Vouloir saisir les émotions 'à la deuxième personne' s'inscrit dans cette démarche de recherche en mettant en avant les compétences pratiques des acteurs, c'est-à-dire leur capacité de percevoir et d'interpréter les expressions émotionnelles.

Toutefois, rendre compte d'une part des capacités perceptives et interprétatives des professionnels et d'autre part de l'objet de ces activités perceptives et interprétatives pose des défis méthodologiques.

Deux dispositifs méthodologiques semblent nécessaires et complémentaires: les observations d'audience et les entretiens 'à chaud' avec les professionnels du droit.

Les observations d'audience sont indispensables pour décrire l'activité langagière et corporelle qui rend visible et intelligible l'action judiciaire en train de se faire (Dupret \& Ferrié, 2006: 113). L'enjeu est de parvenir à rendre compte de la 'natural accountability' - qui est largement non-problématique - de la scène vécue dans les 'formal renderings' (Garfinkel, 2002). Or, il existe un 'fossé entre la transformation d'activités accomplies, incarnées et vécues localement en documents textuels désengagés' (Dupret, 2014: 77). Pour combler ce fossé, plusieurs techniques peuvent être mises en place.

L'enregistrement audio des audiences est utile non seulement pour capter les échanges de paroles mais également pour décrire les intonations, les variations de la voix, le débit de paroles, etc. afin que la retranscription des échanges soit la plus 'vivante' possible. Les descriptions peuvent alors mentionner l'empathie du juge, le sarcasme à peine contenu d'une intervention ou le ton outré d'une réplique. 
Notons que l'enregistrement vidéo ${ }^{17}$ (Aranguren \& Tonnelat, 2014; Velkovska \& Zouinar, 2102), bien qu'il soit proscrit dans les audiences - il ne peut donc être mis en œuvre sur un tel terrain - offre un dispositif méthodologique très intéressant. Il permet une retranscription minutieuse des moindres mouvements qui pourraient échapper à la prise de note en situation et enrichir l'ethnographie. L'enregistrement vidéo peut également être mis en place pour permettre aux professionnels de commenter leurs propres pratiques en offrant des repères aux comptes rendus des acteurs selon la méthode des cours d'action (Theureau, 2010).

Pour parvenir à décrire les nombreux indices non-verbaux, l'ethnographe essaie uniquement de prendre note des mouvements du corps, postures, mimiques faciales, regards, gestuelle qu'il observe durant l'audience. L'idéal est de pouvoir observer l'audience à plusieurs chercheurs pour pouvoir se focaliser sur des acteurs différents (justiciables, magistrats et avocats). En effet, s'il est intéressant de relever les expressions faciales de la personne qui parle, il est tout aussi intéressant de noter la manière dont sont reçues ces expressions. Il est donc important de pouvoir consigner les réactions des destinataires. Il n'est pas possible de décrire toutes les expressions non-verbales de tous les acteurs. L'observateur sélectionne les moments qui l'ont frappé. Il décrit souvent les événements marquants car ils sortent de l'ordinaire ou sont particulièrement chargés émotionnellement. Ces notes ne visent pas l'objectivité. Elles consignent les perceptions du sociologue. Elles seront par la suite soumises aux professionnels du droit lors d'entretiens 'feedback', directement après l'audience observée afin que ce matériau de première main soit relu et interprété avec les yeux experts de l'avocat et du magistrat. Elles fournissent des points de repère pour rappeler certains moments saillants de la scène vécue et les commenter.

Les techniques liées à l'observation ont pour ambition de faire apparaître les expressions émotionnelles observables par tout participant au procès, qu'il soit professionnel ou profane. Cependant, certains signes sont uniquement perceptibles par les professionnels. En outre, les procédures d'interprétation ne sont que rarement observables. Dès lors, si l'observation d'audiences est nécessaire, elle parait insuffisante. Les spécificités de l'action judiciaire d'une part et l'attention donnée aux perceptions et interprétations d'autre part obligent à compléter l'observation des audiences par des entretiens 'à chaud' avec les professionnels du droit.

Les professionnels du droit, comme c'est le cas de tous les professionnels membres d'une communauté de pratiques, partagent un certain nombre d'attentes d'arrière-plan, de compétences pratiques et de savoirs tacites qui leur permettent de se comprendre et de se coordonner dans les cours d'action. Or, le sociologue non-membre qui n'a pas les compétences requises se trouve face à un matériau qui lui est en partie inaccessible. En effet, les avocats ne plaident pas de la même manière devant tous les juges. Ils se basent sur leur expérience pour adapter leur plaidoirie. Ils se montreront vindicatifs devant certains juges et plus doux devant d'autres. Ces variations, qui sont perceptibles pour des avocats habitués à plaider ensemble, passent inaperçues pour un observateur extérieur, fût-il juriste.

Une autre spécificité de l'action judiciaire tient à son caractère écrit et processuel. L'audience prend place dans une procédure judiciaire qui organise l'échange écrit de documents. Cela a des implications pour les échanges de paroles. Ainsi la compréhension des conversations se fait en contexte. Or, le contexte renvoie non seulement au 
contexte d'énonciation, mais également au contexte de l'affaire, c'est-à-dire aux documents échangés avant l'audience (échange de courriers, échange de conclusions) ou aux pièces qui se trouvent dans le dossier. Lors des audiences, les acteurs parlent de beaucoup de choses sans les mentionner expressément, et ces choses sont comprises parce qu'elles font implicitement référence à ce que tout le monde sait de l'affaire. L'avocat qui plaide n'évoque pas certains détails car tous les acteurs au procès les connaissent. Or, ce savoir lié à l'affaire échappe à l'observateur, qui n'a pas pu prendre connaissance du dossier avant l'audience.

L'observateur est capable de saisir certaines expressions émotionnelles, mais qu'en est-il de leur perception et de leur interprétation en action? Certaines scènes sont facilement descriptibles car les professionnels réagissent ouvertement à ces expressions émotionnelles. C'est le cas de l'avocat qui pose la main sur l'épaule de son client trop bavard ou trop énervé; du magistrat qui, jusqu'alors plongé dans son dossier, relève soudain la tête et fixe l'avocat qui plaide; de l'avocat ennuyé par une question du juge, qui fouille fébrilement dans son dossier. Mais en dehors de ces réactions visibles, il est difficile d'accéder directement aux perceptions et interprétations plus contenues.

Pour parvenir à saisir les significations attribuées à ces comportements expressifs, il nous paraît indispensable de réaliser des entretiens à chaud avec les professionnels du droit. Les ont-ils remarqués? Comment les ont-ils perçus? Qu'est-ce qui dans la scène d'action a constitué des appuis pratiques pour mener à bien leur activité?

Alors par contre pendant la plaidoirie, moi j'essaie toujours d'un peu déceler chez les magistrats voir s'ils ont l'attention captée, voir s'ils tiquent sur quelque chose, s'ils ont des mouvements de visage. Je ne sais pas si vous avez remarqué, mais je regarde toujours, je n'aime pas plaider avec des notes même si je les avais devant moi, pour voir si j'arrive à déceler quoi que ce soit ...et quand le président a commencé à me poser une ou deux questions, je me suis dit: 'ah, il est peut-être en train de se dire que c'est pas suffisamment grave'. (Avocat suite à sa plaidoirie au tribunal du travail)

Dans l'extrait d'entretien cité ci-dessus, qui a été réalisé directement après une audience au tribunal du travail en matière de licenciement des travailleurs protégés, l'avocat confie qu'il essaie de décoder dans l'attitude du magistrat des signes susceptibles d'infléchir sa plaidoirie. Il fait référence au moment où le juge lui pose des questions. 'Je me suis dit' indique l'activité interprétative de l'avocat. Ce feedback a permis d'enrichir le compte rendu de l'audience observée et de mettre en lien les tours de paroles et les procédures pratiques d'interprétation de l'avocat.

Cette méthodologie est peu orthodoxe au regard des acquis ethnométhodologiques selon lesquels l'action est 'accountable', les comptes rendus rétrospectifs n'étant pas nécessaires pour comprendre l'action. Il est vrai que l'action intelligente se déroule pour une bonne part sans être formulée. La compréhension s'opère toujours par rapport à un arrièrefond fait de ce que l'on tient pour acquis, de ce sur quoi l'on s'appuie. Cet arrière-fond incorpore véritablement une compréhension: il consiste en une appréhension des choses qui, quoiqu'implicite, peut nous permettre de formuler des raisons et des explications si on nous met au défi d'en donner (Taylor, 1995: 557). Autrement dit, si nos actions sont le plus souvent non explicitées, elles sont explicitables. Il appartient au sociologue de rendre explicite l'implicite en mettant en avant les savoirs professionnels incorporés, les 
procédures d'interprétation ordinaires, les connaissances de sens commun. Les entretiens permettent alors d'éclairer les zones restées sombres lors des observations par manque de compétences pratiques du sociologue non-membre.

Ayant participé à la même audience, le sociologue et le professionnel du droit peuvent confronter leurs perceptions et interprétations des moments forts, des indices corporels, des émotions exprimées. Les notes et observations que le sociologue soumet au professionnel du droit permettent une remise en situation et un processus d'autoconfrontation (Theureau, 2010). Ainsi replacé dans la scène d'action par l'intermédiaire des rappels d'événements vécus, le magistrat et l'avocat peuvent plus facilement évoquer quels étaient les appuis sensibles qui ont permis la coordination. Il importe de pouvoir avoir accès à ce que les professionnels ont vu, ont entendu, ont remarqué, ont senti. Revenir avec les professionnels sur ces activités permet d'avoir accès à ce matériau peu observable. Ces entretiens ambitionnent de récolter des activités pratiques et non des récits qui mettent en avant une action lissée par une rationalité livrée a posteriori. Pour tenter de réduire l'écart entre les activités 'en train de se faire' observables et leur compte rendu, il convient d'éviter de poser des questions qui amènent le magistrat ou l'avocat à livrer des 'réflexions abstractives' (Barthélemy \& Quéré, 2007: 17). Les questions doivent faire référence à l'audience vécue, aux opérations situées, aux méthodes pratiques, aux émotions observées, aux indices concrets, aux événements marquants, etc.

\section{Conclusion}

Accorder une place aux émotions dans l'action judiciaire permet de saisir le droit tel qu'il se fait et tel qu'il se vit. Cela nécessite d'aborder les émotions de manière située plutôt que de réifier le phénomène émotionnel en un catalogue abstrait. Cela nécessite aussi d'adapter notre arsenal conceptuel afin d'intégrer le phénomène émotionnel au cœur de l'agir. Plutôt que de tenter de situer les émotions sur les grandes dichotomies (corps-esprit, culture-nature, raison-passion), nous proposons de regarder ce que les expressions émotionnelles font faire aux acteurs qui les perçoivent: Comment sont-elles repérées dans l'interaction, comment sont-elles interprétées en situation et comment interviennent-elles dans la compréhension du monde? Ces expressions émotionnelles apparaissent comme des ressources situationnelles que les avocats et les juges utilisent pour orienter leurs activités pratiques.

Cependant, si les émotions participent à l'action en donnant une profondeur phénoménale ${ }^{18}$ aux cours d'action, elles ne peuvent être vues comme le moteur de l'action ou comme une composante autonome de l'action. Elles en font partie, comme en font partie le contexte ou les documents. Il s'agit moins d'étudier les émotions à la première personne, le 'je' qui ressent les émotions, qu'à la deuxième personne, le 'tu' chez qui l'on perçoit l'émotion. Apparaissent alors les concepts de sens, d'expérience et de connaissances incorporées, qui supplantent les concepts de rationalité, de cogito et de volonté.

La prise en compte des expressions émotionnelles dans l'activité judiciaire nous invite à repenser le rapport entre le sensible et l'intelligible (Thibaud, 2002), ce qui va à rebours des approches dominantes, qui font ressortir la signification des phénomènes sociaux, l'épure de toutes ses empreintes sensorielles (Sansot, 1986: 5). Le sens ne va pourtant pas sans les sens, ni l'intelligible sans le sensible (Quéré, 1999). La perception, 
en ce qu'elle renvoie à ces deux types d'activité, est par conséquent centrale pour rendre compte de la place des expressions émotionnelles dans l'action.

\section{Financement}

Cette étude n'a bénéficié d'aucune source de financement spécifique d'un commanditaire du secteur public, privé ou à but non lucratif.

\section{Notes}

1. 'Le droit ne connait pas la colère.'

2. Le monde du droit a, de tout temps, été traversé de notions affectives, que l'on pense à l'intime conviction du juge, aux sentiments d'injustice, à la notion de culpabilité. Depuis une trentaine d'années, le droit positif compte certaines législations plus ouvertes à des considérations éthiques. On pense à la loi du 11 juin 2002 relative à la protection contre la violence et le harcèlement moral ou sexuel au travail (M.B. 22 juin 2002), la loi du 28 mai 2002 relative à l'euthanasie (M.B. 22 juin 2002) ou l'attention portée à l'intérêt supérieur de l'enfant (Lebreton, 2003). Ces concepts juridiques renvoient à une qualification qui ne va pas sans porter une forte charge émotionnelle. En outre, le procès pénal voit son déroulement impacté par la place grandissante que prennent les victimes (Hammer et al., 2007), qui souhaitent voir leur souffrance reconnue (Honneth, 2000) publiquement et juridiquement.

3. La praxéologie du droit (Dupret, 2006) est un programme de recherche qui s'intéresse aux procédures par lesquelles les professionnels du droit interprètent les circonstances dans lesquelles ils se trouvent, et conçoivent et mettent en œuvre des modes d'action (Heritage, 1984). L'action est envisagée comme un accomplissement pratique multimodal (Mondada, 2012). Sous ce label, on regroupe les études qui se rattachent à l'ethnométhodologie, c'est-àdire l'étude des manières (des méthodes) par lesquelles les gens (les membres d'un groupe, d'une ethnie) donnent du sens à leur monde d'action, s'orientent vers ce monde et le pratiquent quotidiennement et routinièrement. L'ambition de ce programme de recherche est de respécifier les études juridiques en saisissant le droit comme un accomplissement pratique: il s'agit de comprendre comment s'organise pratiquement l'activité professionnelle des juristes. Comment s'ordonnent et se dotent de sens les activités juridiques et juridictionnelles.

4. 'En adoptant ce point de vue, l'individu confère de la sorte à son interlocuteur un pouvoir de sanctionner un coup (énoncé ou action) qui serait incorrectement joué dans le cours de l'interaction qui les réunit' (Ogien, 2009: 506).

5. Le réalisme juridique américain et sa 'breakfast jurisprudence' incarnent dans le domaine du droit de manière quelque peu caricaturale le rôle des émotions ressenties par le juge dans le processus décisionnel.

6. Martha Nussbaum (1995) s'est penchée sur le lien existant entre les émotions et la perception. Elle reprend l'idée sartrienne selon laquelle les émotions sont des façons - certaines façons particulières - de percevoir les choses (Nussbaum, 1995: 25). 'L'émotion est une certaine manière d'appréhender le monde' (Sartre, 1995: 71). La manière de percevoir est propre à chacun car on rattache cette perception à notre expérience singulière. En outre, les émotions sont cognitives dans le sens où elles sont liées à certaines croyances. 'La colère, par exemple, exige la croyance que l'action intentionnelle d'une autre personne m'a nui, à moi ou à quelque chose qui est important pour moi. Si cette croyance complexe en vient à cesser de me paraître vraie, sous quelque aspect significatif que ce soit - si je change d'idée sur celui qui m'a fait du mal, sur le fait que c'était intentionnel, ou sur le fait que ce qui s'est passé était effectivement du mal - on peut s'attendre à ce que ma colère se calme ou change son cours en conséquence' (Nussbaum, 1995: 25). Cette manière de ramener les émotions dans le giron 
de la cognition a l'avantage de mettre en doute la pertinence des critiques qui considèrent les émotions comme simplement irrationnelles car étrangères au jugement et à la cognition. Cependant, partir des émotions ressenties par l'acteur et examiner le lien que les unit à certaines croyances et expériences personnelles prive le sociologue de l'ancrage social des émotions, qui demeurent un phénomène intime et personnel.

7. Les modèles dominants de l'action, qu'il s'agisse de l'action rationnelle (Weber, 1971) ou de l'action normative (Parsons), s'appuient sur une conception du sujet moderne regardé comme un individu pensant - relativement - libre et responsable de ses jugements (Joas, 1999). Cette manière de concevoir l'homme moderne est imprégnée de la distinction cartésienne du corps et de l'esprit. 'Chacun d'entre nous se voit appelé à devenir un esprit pensant responsable, autonome dans ses jugements' (Taylor, 1995: 559). L'esprit apparaît comme le siège cognitif de ce sujet rationnel, le corps étant perçu comme un instrument au service de la volonté consciente de l'individu.

8. On retrouve le modèle mécanique dans certains travaux neurobiologiques et neurochimiques qui font du corps humain un simple récepteur de stimuli neuronaux.

9. L'indexicalité, selon Coulon (2007 [1987]: 27), désigne 'l'incomplétude naturelle des mots, qui ne prennent leur sens "complet" que dans leur contexte de production, que s'ils sont "indexés" à une situation d'échange linguistique'.

10. Nous percevons directement le dédain, la colère ou la joie dans les interactions. Comme le souligne Quéré (1999: 311), qui reprend les enseignements de Wittgenstein sur le "voir comme', nous percevons directement les choses avec leur signification.

11. Comme le soulignent Coulter et Parsons, le caractère remarquable d'un élément qui n'est pas censé l'être peut avoir une résonnance personnelle, l'élément remarqué renvoyant à des expériences vécues précédemment. 'Remarquer quelque chose qui n'est pas conventionnellement "remarquable" dans un contexte peut donner lieu à un compte rendu, exigé ou spontané, et ce compte rendu peut avoir une forme qui indique une pertinence strictement personnelle (un souvenir, une réaction émotive, etc.)' (Coulter \& Parsons, 2002: 235).

12. En Belgique, le CPAS (Centre Public d'Action Sociale) est une institution publique qui octroie le revenu d'intégration sociale afin d'offrir des revenus minimum à l'ensemble de la population. Ce revenu d'intégration est toutefois soumis à une série de conditions.

13. Précisons que ce cas est paradigmatique. Les magistrats sont généralement en mesure de trouver la solution juridique à la lecture du dossier, et l'audience vient confirmer - et parfois infirmer - leur première impression. Il existe cependant des affaires où les choses ne sont pas claires et où le juge hésite. L'audience offre alors des indices qui nourriront le processus décisionnel.

14. Le processus interprétatif à l'œuvre dans le raisonnement judiciaire pratique fait écho à la méthode documentaire d'interprétation (Garfinkel, 2007: 152ss) qui consiste à traiter une apparence comme l'illustration d'une structure sous-jacente dont l'existence est présupposée. Cette méthode d'interprétation est dynamique car elle s'appuie sur un aller-retour constant entre la situation d'ensemble et les détails particuliers qui la composent (Thibaud, 2002: 30). 'Non seulement le pattern sous-jacent a été tiré du cours des évidences documentaires individuelles, mais en outre celles-ci, à leur tour, ont été interprétées sur la base de "ce qui est connu" et de ce qui pouvait être anticipé des patterns sous-jacents' (Garfinkel, 2007: 102-103).

15. Watson (1989: 97) attire l'attention sur le risque 'd'ironisation' qui peut découler de l'utilisation de la métaphore du jeu goffmanienne.

16. 'L'ethnométhodologie et analyse de conversation rejettent cette forme d'ironie qui caractérise la démarche sociologique dans sa grande majorité, quand elle place le chercheur en "surplomb" de la réalité sociale et juridique, lui octroyant de la sorte un monopole de la 
compréhension de ce qui se passe "vraiment" et que l'acteur ne saurait voir de lui-même' (Dupret, 2006: 8).

17. Le dispositif méthodologique initialement envisagé était le suivant: dans un premier temps, nous souhaitions filmer l'audience et demander dans un second temps aux avocats et magistrats présents de commenter certains passages afin de recueillir leur interprétation de la scène vécue.

18. La profondeur phénoménale renvoie à ce qui fait la chaire du social, au fait sensible et qui est souvent mis de côté lors des comptes rendus sociologiques.

\section{Références}

Aranguren M, Tonnelat S (2014) Emotional transactions in the Paris subway: Combining naturalistic videotaping, objective facial coding and sequential analysis in the study of nonverbal emotional behaviour. Journal of Nonverbal Behavior (décembre).

Bandes S (1996) Empathy: Narrative and victim impact statements. University of Chicago Lew Review 63(2): 361-412.

Bandes S (1999) The Passions of Law. New York, NY: New York University Press.

Barbot J, Dodier N (2014) Que faire de la compassion au travail? La réflexivité stratégique des avocats à l'audience. Sociologie du travail 56: 365-385.

Barthelemy M, Quéré L (2007) L'argument ethnométhodologique, introduction à H. Garfinkel. Recherches en ethnométhodologie. Paris: PUF-Quadrige, 9-44.

Ben-Ze'ev A (1997) Emotions and morality. Journal of Value Inquiry 31: 195-212.

Bessy C, Châteaureynaud F (1995) Experts et faussaires: Pour une sociologie de la perception. Paris: Métaillé.

Cislaru G (2008) L'intersubjectivation des émotions comme source de sens: expression et description de la peur dans les écrits de signalement. Carnets $d u$ Cediscor. http://cediscor.revues. org/195.

Coulon A (2007) L'ethnométhodologie. Paris: PUF (Que sais-je?). (1 $1^{\text {re }}$ éd. 1987)

Coulter J, Parsons ED (2002) Praxéologie de la perception: orientations visuelles et action pratique. In: Thibaud JP (éd.) Regards en action: Ethnométhodologie des espaces publics. Grenoble: A la croisée, 213-239.

Damasio AR (1995) L'erreur de Descartes: La raison des émotions. Paris: Odile Jacob.

Dupret B (2006) Droit et sciences sociales. Paris: Armand Colin.

Dupret B (2014) Le Code en tant qu'accomplissement pratique. Respécification ethnométhodologique et cas d'étude égyptien. Tracés, Revue des Sciences humaines 27: 74-92.

Dupret B, Ferrié JN (2006) Morale ou nature: Négocier la qualification de la faute dans une affaire égyptienne d'homosexualité. Négociations 2(2): 41-57.

Flückiger A (2009) Pourquoi respectons-nous la soft law? Le rôle des émotions et des techniques de manipulation. Revue européenne des sciences sociales 47(144): 73-103.

Game A (1997) Sociology's emotions. Canadian Review of Sociology 34: 385-399.

Garfinkel H (2002) Ethnomethodology's Program: Working out Durkheim's aphorism. Oxford: Rowman \& Littlefield.

Garfinkel H (2007) Recherches en ethnométhodologie. Paris: PUF (Quadrige).

Gross J (2013) Handbook of Emotion Regulation. New York: The Guilford Press.

Hammer R, Hauger S, Languin N (2007) Emotions et droit: entre affinités et tensions. Sciences croisées, revue en ligne de l'école doctorale. Cognition, langage, éducation no. 1.

Heritage J (1984) Garfinkel and Ethnomethodology. Cambridge: Polity Press.

Hochschild AR (2003) Travail émotionnel, règles de sentiments et structure sociale. Travailler 1(9): 19-49. 
Honneth A (2000) La lutte pour la reconnaissance, trad. Rusch P. Paris: Cerf.

Joas H (1999) La créativité de l'agir. Paris: Cerf.

Joas H (2001) La créativité de l'agir. In: Baudouin J-M, Friedrich J (éds) Théories de l'action et éducation. Bruxelles: De Boeck Université, 27-43.

Karstedt S (2002) Emotions and criminal justice. Theoretical Criminology 6: 299-317.

Kelsen H (1999) Théorie pure du droit. Paris: LGDJ.

Le Breton D (2004) Les passions ordinaires: Anthropologie des émotions. Paris: Payot et Rivages (Petite bibliothèque Payot).

Lebreton G (2003) Le droit de l'enfant au respect de son 'intérêt supérieur': Critique républicaine de la dérive individualiste du droit civil français. Cahiers de Recherche sur les Droits Fondamentaux 2: 77-86.

Lynch M, Haney C (2014) Emotion, authority, and death: (Raced) negotiations in mock capital jury deliberations. Law and Social Inquiry 40(2): 377-405.

Mondada L (2012) Organisation multimodale de la parole en interaction: Pratiques incarnees d'introduction des référents. Langue française 3(175): 129-147.

Nussbaum M (1995) Les émotions comme jugement de valeur. In: Paperman P, Ogien R (éds) $L a$ couleur des pensées. Raisons pratiques no. 6. Paris: Ed. de l'EHESS, 19-33.

Ogien A (2007) Les formes sociales de la pensée: La sociologie après Wittgenstein. Paris: Armand Colin.

Ogien A (2009) Du sens commun comme d'une sorte de faculté de juger. In: Gautier C, Laugier S (éds) Normativités du sens commun. Paris: PUF, 445-463.

Ogien R, Paperman P (éds) (1995) La couleur des pensées: Sentiments, émotions, intentions. Raisons pratiques, no. 6. Paris: Ed. de l'EHESS.

Papaux A (2009) Un droit sans émotions. Iram non novit ius: esquisse des rapports entre science et droit. Revue européenne des sciences sociales 47(144): 105-119.

Paperman P (1995) La question des émotions: du physique au social. L'Homme et la société 116: $7-17$.

Paperman P (2000) La contribution des émotions à l'impartialité des décisions. Informations sur les sciences sociales 39(1): 29-73.

Pizzaro D (2000) Nothing more than feelings? The role of emotions in moral judgment. Journal of the Theory of Social Behaviour 30: 355-375.

Quéré L (1999) Action située et perception du sens. In: de Fornel M, Quéré L (éds) La logique des situations. Raisons pratiques, no. 10. Paris: Ed. de l'EHESS, 301-338.

Quéré L (2012) Le travail des émotions dans l'expérience publique: Marées vertes en Bretagne. In: Cefaï D, Terzi C (éds) L'expérience des problèmes publics. Raisons pratiques no. 22. Paris: Ed. de l'EHESS: 135-162.

Sansot P (1986) Les formes sensibles de la vie sociale. Paris: PUF (La politique éclatée).

Sartre JP (1995) Esquisse d'une théorie des émotions. Paris: Le livre de poche.

Scherer KL (2011) On the rationality of emotions; or, When are emotions rational? Social Science Information 50(3/4): 330-350.

Sudnow D (1965) Normal crimes: Sociological features of the penal code in a public defender office. Social Problems 12(3): 255-276.

Taylor C (1995) Suivre une règle. Critique 579-580 (septembre): 554-572.

Theureau J (2010) Les entretiens d'autoconfrontation et de remise en situation par les traces matérielles et le programme de recherche 'cours d'action'. Revue d'anthropologie des connaissances 4(2): 287-322.

Thibaud J-P (2002) Regards en action: Ethnométhodologie des espaces publics. Grenoble: A la croisée. 
Travers M (2001) Ethnométhodologie, analyse de conversation et droit. Droit et société 48: $349-366$.

Turner JH (2009) The sociology of emotions: Basic theoretical arguments. Emotion Review 1(4): 340-354.

Velovska J, Zouinar M (2012) Jugements et émotions dans les interactions institutionnelles. ethnographiques.org. Revue en ligne des sciences humaines et sociales 25 (décembre).

Watson R (1995) Angoisse dans la 42 ème rue. In: Ogien R, Paperman P (éds) La couleur des pensées: Sentiments, émotions, intentions. Raisons pratiques, no. 6. Paris: Ed. de l'EHESS.

Watson R (1989) Le travail de l'incongruité. In: Castel R, Cosnier J, Joseph I (éds) Le parler frais d'Erving Goffman. Paris: Les Editions de Minuit, 83-99.

Weber M (1971) Economie et société. Paris: Plon. 